\title{
Adding Psychotherapy to the Naltrexone Treatment of Alcohol Use Disorder: Meta-analytic Review
}

Rizwan Ahmed ${ }^{1}$, Vijaya Padma Kotapati ${ }^{2}$, Ali M. Khan ${ }^{3}$, Nuzhat Hussain ${ }^{4}$, Mudasar Hussain ${ }^{5}$, Sara Dar 6 , Jeevan Kumar ${ }^{7}$, Gulshan A. Begum ${ }^{2}$, Michael Esang ${ }^{8}$, Navjot Brainch ${ }^{9}$, Saeed Ahmed ${ }^{8}$

1. Psychiatry, Liaquat College of Medicine \& Dentistry, Karachi, PAK 2. Psychiatry, Manhattan Psychiatric Center, New York, USA 3. Psychiatry Resident, University of Texas Rio Grande Valley, Harlingen, Texas, USA 4. Psychiatry, Penn State University College of Medicine, Pennsylvania, USA 5. Psychiatry, NYU Langone Medical Center, New York, USA 6. Psychiatry, Brigham and Women's Hospital, Boston, USA 7. Psychiatry, Bolan Medical College, Quetta, PAK 8. Behavioral Health Sciences, Nassau University Medical Center, East Meadow, USA 9. Psychiatry, Maimonides Medical Center, Brooklyn, USA

Corresponding author: Vijaya Padma Kotapati, padmakotapati89@gmail.com

\section{Abstract}

\section{Background}

It remains unclear if naltrexone combined with psychotherapy is superior to naltrexone alone in treating alcohol use disorders (AUD). The current meta-analysis examined the hypothesis that psychotherapy is a significant moderator that influences AUD-related outcomes and that naltrexone combined with psychotherapy is associated with significantly better AUD-related outcomes than naltrexone alone.

\section{Methods}

A total of 30 studies $\left(\mathrm{N}_{\text {naltrexone }}=2317 ; \mathrm{N}_{\text {placebo }}=2056\right)$ were included. Random effects model metaanalyses were carried out for each of the studied outcomes. Subsequently, the random effects model pooled estimates from studies with and without psychotherapy were compared using a Wald test. A mixed-effect model, incorporating psychotherapy as a moderator, was used to examine the impact of psychotherapy on treatment outcomes.

\section{Results}

Naltrexone had a significant treatment effect on abstinence relapse and Gamma-Glutamyl Transferase levels, but not cravings. The pooled estimates for studies with and without psychotherapy were not significantly different for any of the studied outcomes. Psychotherapy was not a significant moderator in the mixed effects models for any of the studied outcomes.

Received 07/09/2018 Review began 07/25/2018 Review ended 08/04/2018 Published 08/06/2018

() Copyright 2018 Ahmed et al. This is an open access article distributed under the terms of the Creative Commons Attribution License CC-BY 3.0 , which permits unrestricted use, distribution, and reproduction in any medium, provided the original author and source are credited.

\section{Conclusions}

Naltrexone treatment is efficacious in reducing alcohol consumption, but not reducing cravings. Adding psychotherapy on top naltrexone did not result in any significant additional benefit for AUD patients.

Categories: Psychiatry, Psychology, Miscellaneous

Keywords: alcohol use disorder, naltrexone, psychotherapy, abstinence, relapse, gamma-glutamyl transferase, cravings

\section{Introduction}

Alcohol use disorders (AUD) are debilitating psychiatric illnesses characterized by the abuse of and dependence on alcohol. The lifetime prevalence of AUD is estimated to be at 29.1\%. AUD bring about devastating consequences to the individual. They are associated with a high disease-related burden chronic alcohol consumption is causally implicated in cancer, cardiovascular disease, liver cirrhosis, and injury. On top of that, an estimated $3.8 \%$ of deaths and $4.6 \%$ of disability life years are attributable to alcohol. At the societal level, the economic costs associated with AUD amounts to at least $1 \%$ of the gross national product in high and middle-income countries. Given such consequences, the need to treat AUD cannot be understated. Ever since naltrexone was approved as a treatment for alcohol dependence in 1994 by the U.S. Federal Drug Administration, it has been widely used as a first-line pharmacological treatment for AUD. As a $\mu$-opioid receptor antagonist, naltrexone blocks alcohol-related dopamine release in the mid-brain reward system, thereby reducing the reward and reinforcement associated with alcohol consumption.

Many controlled trials have been carried out over the past three decades to examine the efficacy of 
naltrexone on various AUD-related outcomes and several meta-analyses have been carried out to synthesize the results of these studies. These meta-analyses have generally indicated moderate to strong efficacy in treating AUD with naltrexone. However, these meta-analyses included studies that carried out various psychotherapies to augment the naltrexone treatment of AUD. Two studies [1, 2] had previously examined the effect of adding psychotherapy to naltrexone treatment in treating AUD, within-study, and concluded that adding psychotherapy to the naltrexone treatment of AUD did not result in significantly better outcomes. Apart from these two studies, it remains largely unclear if the significant treatment effect is primarily attributed to naltrexone alone or the combination of naltrexone and psychotherapy. Such information would be useful to optimize existing naltrexone interventions on AUD.

To this end, in addition to examining the overall efficacy of naltrexone on AUD, we also examined the impact of psychotherapy in two different ways. First, we compared the pooled estimates between studies with and without psychotherapy; then we examined the moderation effect of psychotherapy on the treatment outcomes. As with previous meta-analyses on AUD intervention studies, we hypothesized that the meta-analysis would yield significant effect sizes favoring the use of naltrexone on AUD. Additionally, based on a previous meta-analysis comparing between pharmacological-only treatment and combined psychotherapy and pharmacological treatment, we hypothesized that studies of naltrexone treatment combined with psychotherapy yield significantly better AUD-related outcomes relative to those of naltrexone alone studies.

\section{Materials And Methods Data sources and extraction}

Using the Preferred Reporting Items for Systematic Reviews and Meta-Analyses (PRISMA) methodology, a search for relevant published literature was carried out on PubMed, Medline and Cochrane using the keywords "naltrexone”, "low dose naltrexone”, "high dose naltrexone”, "naltrexone alcohol”, "naltrexone psychotherapy”. This search started on 2nd February 2017 and was last carried out on 30th March 2017. The reference lists of relevant studies were also manually searched for additional studies. Only articles in English were retrieved. We included studies of controlled trials involving naltrexone treatment on AUD. Specifically, these studies must be of an independent group's pretest-posttest design. There were no restrictions on patients' age or level of alcohol use. Researches that studied AUD-related outcomes using experimental/laboratory paradigms were not included in the meta-analysis.

Means and standard deviations (SD) as well as event counts of the trial outcomes, and information pertaining to the participants (sample size, age, sex, diagnosis), and trial-design (naltrexone dose, duration and psychotherapy) for each study were entered into a structured data abstraction form by the two research assistants. If there were discrepancies among both of them, the entered data was checked against the source. In cases, where there were multiple naltrexone treatment groups (i.e., different dosages), these means, SDs and event counts of these groups were combined using the formulae for combining groups. Due to the multitude of outcomes studied among the retrieved studies, only data from outcomes, which have been reported by at least four studies in each of the with and without psychotherapy subgroups, were extracted. For the purpose of the current study, we define psychotherapy as non-pharmacological treatment, such as individual and group therapy, psychoeducation, counseling and skills training, of at least two sessions, aimed at modifying cognition and/or behaviors associated with alcohol consumption. Hence, interventions that focused solely on adherence or medication compliance are not considered as psychotherapy. The flow chart for the selection of studies and data extraction is shown in Figure 1. 


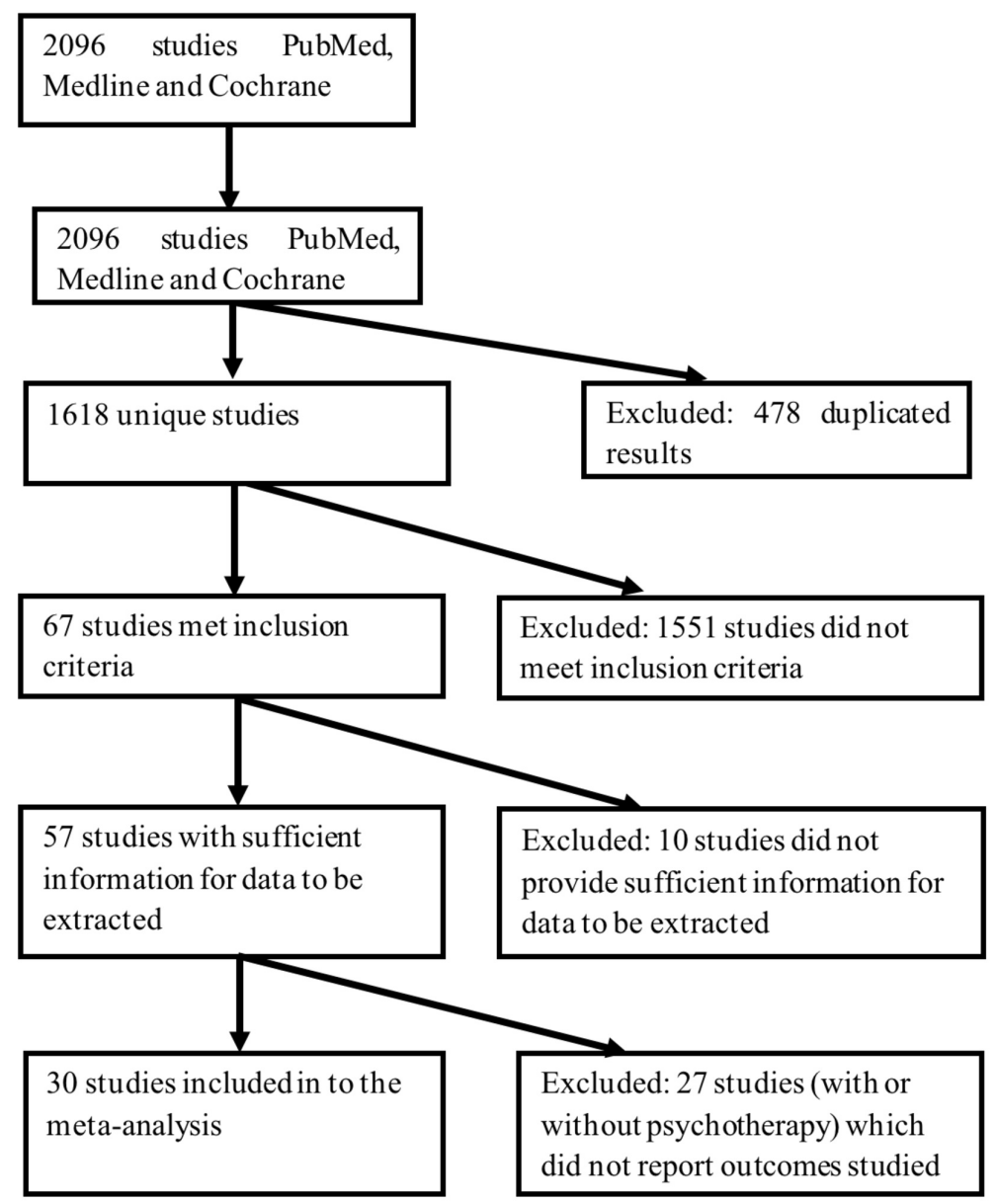

FIGURE 1: Selection of studies.

Data Synthesis

For outcomes involving dichotomous variables only at post-treatment, such as the number of participants who relapsed to heavy drinking or remained abstinent, the log Odds Ratios (OR) and their respective sampling variance were calculated using the escalc function in the metafor package in $\mathrm{R}$. As for continuous outcomes studied both at baseline and post-treatment such as Gamma-Glutamyl Transferase (GGT) levels and cravings, the raw score single group pretest-posttest standardized mean difference (SMD) for both the treatment and placebo groups was computed separately using Formula A1. Following which, the placebo group's SMD was subtracted from that of the treatment group to obtain the overall SMD for a particular studied outcome in the study. The sampling variances of the treatment and placebo subgroups were then summed to obtain the overall sampling variance for that outcome in the study.

These SMDs and ORs were then pooled and meta-analyzed using random effects models (REM), to allow the effect sizes to vary across studies. The direction of the effect sizes was coded in a manner that larger positive effect sizes correspond to larger positive differences (i.e., treatment - placebo) in raw scores. The Wald test was used to compare the pooled estimates between studies with and without psychotherapy. Next, a mixed effects model was meta-analyzed with psychotherapy (coded dichotomously for presence or absence) included as a moderator. Heterogeneity was measured using the Q statistic; a significant Q statistic suggests that the variability among the effect sizes is larger than what is expected from subject sampling error alone. In addition, trim and fill analyses were carried out to assess whether publication bias had significantly influenced the aggregated effect sizes. Specifically, the trim and fill analyses determine whether there are missing effect sizes, if so impute the missing effect sizes, and then recalculate the aggregated effect sizes. Follow-up leave-one-out analyses were carried out to assess the robustness of the results. These analyses were carried out using the metafor package in R 3.4.0.

\section{Results}

The study and participant characteristics of all included studies are presented in Table 1 and Table 2, 
respectively. At least four studies in each of the with or without psychotherapy subgroups had reported data on abstinence, relapse, cravings, and GGT; these variables were then included in the meta-analyses. The results of the meta-analysis are shown in Table 3. The forest plots for the REMs of all studies are presented in Figures 2-5. Significant pooled estimates were obtained for the REM involving studies with psychotherapy for abstinence and relapse and all studies, for abstinence, relapse, and GGT. The significant negative effect sizes for relapse and GGT, and positive effect size for abstinence indicated that participants in the naltrexone treatment conditions had significantly better outcomes in these areas, relative to those in the placebo group. With the exception of a significant intercept in the mixed effect model (MEM) for relapse, none of the parameters in the MEMs was significant for any of the studied outcomes. Neither were the effect sizes in any of the craving meta-analyses statistically significant. Finally, none of the Wald test statistics was significant, suggesting that the pooled estimates of studies with and without psychotherapy were not significantly different.

\begin{tabular}{|c|c|c|c|c|c|c|}
\hline \multicolumn{2}{|l|}{ Study } & Psychotherapy & Treatment dosage & $\begin{array}{l}\text { Trial } \\
\text { duration }\end{array}$ & Relapse definition & $\begin{array}{l}\text { Measures } \\
\text { of } \\
\text { cravings }\end{array}$ \\
\hline $\begin{array}{l}\text { First } \\
\text { author }\end{array}$ & Year & & & & & \\
\hline $\begin{array}{l}\text { Anton et } \\
\text { al. [3] }\end{array}$ & 2006 & No psychotherapy & 100 mg/day & $\begin{array}{l}16 \\
\text { weeks }\end{array}$ & $\begin{array}{l}\geq 4 \text { drinks/day for } \\
\text { women; } \geq 5 \mathrm{~d} / \mathrm{d} \text { for men }\end{array}$ & OCDS \\
\hline $\begin{array}{l}\text { Anton et } \\
\text { al. [4] }\end{array}$ & 2003 & Cognitive behavioral therapy & 50 mg/day & $\begin{array}{l}12 \\
\text { weeks }\end{array}$ & & $\begin{array}{l}\text { Analog } \\
\text { scale }\end{array}$ \\
\hline $\begin{array}{l}\text { Anton et } \\
\text { al. [3] }\end{array}$ & 2006 & Combined behavioral intervention & 100 mg/day & $\begin{array}{l}16 \\
\text { weeks }\end{array}$ & $\begin{array}{l}\geq 4 \mathrm{~d} / \mathrm{d} \text { for women; } \geq 5 \\
d / d \text { for men }\end{array}$ & OCDS \\
\hline $\begin{array}{l}\text { O'Malley } \\
\text { et al. [5] }\end{array}$ & 1992 & Copıng skills & so mg/day & $\begin{array}{l}12 \\
\text { weeks }\end{array}$ & $\begin{array}{l}\geq 4 \mathrm{~d} / \text { o for women; } \geq 5 \\
\mathrm{~d} / \mathrm{o} \text { for men }\end{array}$ & \\
\hline $\begin{array}{l}\text { O'Malley } \\
\text { et al. [5] }\end{array}$ & 1992 & Supportive therapy & 50 mg/day & $\begin{array}{l}12 \\
\text { weeks }\end{array}$ & & \\
\hline al. [6] & 1997 & Group therapy & 50 mg/day & $\begin{array}{l}12 \\
\text { weeks }\end{array}$ & $\begin{array}{l}\geq 5 \mathrm{~d} / \text { occasion } \text { OR } \geq 5 \\
\mathrm{dd} / \mathrm{w} \text { or } \mathrm{BAC}>100 \\
\mathrm{mg} / \mathrm{dl}\end{array}$ & \\
\hline $\begin{array}{l}\text { Petrakis } \\
\text { et al. [7] }\end{array}$ & 2005 & No psychotherapy & 50 mg/day & $\begin{array}{l}12 \\
\text { weeks }\end{array}$ & & \\
\hline $\begin{array}{l}\text { Volpicelli } \\
\text { et al. [8] }\end{array}$ & 1997 & Relapse prevention & 50 mg/day & $\begin{array}{l}12 \\
\text { weeks }\end{array}$ & $\begin{array}{l}\geq 5 \mathrm{~d} / \mathrm{o} \text { or } \mathrm{BAC}>100 \\
\mathrm{mg} / \mathrm{dl}\end{array}$ & \\
\hline $\begin{array}{l}\text { Davidson } \\
\text { et al. [9] }\end{array}$ & 2004 & Counseling & 50 mg/day & $\begin{array}{l}10 \\
\text { weeks }\end{array}$ & & \\
\hline $\begin{array}{l}\text { Kranzler } \\
\text { et al. [10] }\end{array}$ & 2000 & Relapse prevention psychotherapy & 50 mg/day & $\begin{array}{l}11 \\
\text { weeks }\end{array}$ & & \\
\hline $\begin{array}{l}\text { Monti et } \\
\text { al. [11] }\end{array}$ & 2001 & $\begin{array}{l}\text { Coping skills training and } \\
\text { communication skills training }\end{array}$ & 50 mg/day & $\begin{array}{l}12 \\
\text { weeks }\end{array}$ & $\begin{array}{l}>5 \mathrm{~d} / \mathrm{d} \text { for women; }>6 \\
\mathrm{~d} / \mathrm{d} \text { for men }\end{array}$ & \\
\hline $\begin{array}{l}\text { O'Malley } \\
\text { et al. [12] }\end{array}$ & 2003 & No psychotherapy & 50 mg/day & $\begin{array}{l}24 \\
\text { weeks }\end{array}$ & & OCDS \\
\hline $\begin{array}{l}\text { O'Malley } \\
\text { et al. [12] }\end{array}$ & 2003 & Cognitive behavioral therapy & 50 mg/day & $\begin{array}{l}24 \\
\text { weeks }\end{array}$ & & OCDS \\
\hline $\begin{array}{l}\text { O'Malley } \\
\text { et al. [13] }\end{array}$ & 2008 & No psychotherapy & $\begin{array}{l}1 \times 12.5 \mathrm{mg}+2 \times 25 \mathrm{mg} \\
+50 \mathrm{mg} / \text { day thereafter }\end{array}$ & $\begin{array}{l}16 \\
\text { weeks }\end{array}$ & $\begin{array}{l}\geq 4 \mathrm{~d} / \mathrm{d} \text { for women; } \geq 5 \\
d / d \text { for men }\end{array}$ & $A \cup Q$ \\
\hline Oslin [14] & 2005 & No psychotherapy & 50 mg/day & $\begin{array}{l}12 \\
\text { weeks }\end{array}$ & $\begin{array}{l}\geq 3 \mathrm{~d} / \mathrm{d} \text { for women; } \geq 4 \\
\mathrm{~d} / \mathrm{d} \text { for men }\end{array}$ & \\
\hline $\begin{array}{l}\text { Pettinatı } \\
\text { et al. [15] }\end{array}$ & 2006 & Cognitive behavioral therapy & 50 mg/day & weeks & & \\
\hline $\begin{array}{l}\text { Brown et } \\
\text { al. [16] }\end{array}$ & 2009 & Cognitive behavioral therapy & 50 mg/day & $\begin{array}{l}12 \\
\text { weeks }\end{array}$ & & PACS \\
\hline
\end{tabular}




\section{Cureus}

\begin{tabular}{|c|c|c|c|c|c|c|}
\hline $\begin{array}{l}\text { Anton et } \\
\text { al. [2] }\end{array}$ & 2005 & Cognitive behavioral therapy & $50 \mathrm{mg} /$ day & $\begin{array}{l}12 \\
\text { weeks }\end{array}$ & $\begin{array}{l}\geq 4 \mathrm{~d} / \mathrm{d} \text { for women; } \geq 5 \\
\mathrm{~d} / \mathrm{d} \text { for men }\end{array}$ & \\
\hline $\begin{array}{l}\text { Anton et } \\
\text { al. [2] }\end{array}$ & 2005 & Motivational enhancement therapy & $50 \mathrm{mg} /$ day & $\begin{array}{l}12 \\
\text { weeks }\end{array}$ & $\begin{array}{l}\geq 4 \mathrm{~d} / \mathrm{d} \text { for women; } \geq 5 \\
\mathrm{~d} / \mathrm{d} \text { for men }\end{array}$ & \\
\hline $\begin{array}{l}\text { Kranzler } \\
\text { et al. [17] }\end{array}$ & 2004 & Motivational enhancement therapy & $\begin{array}{l}300 \mathrm{mg}+3 \text { months } x \\
150 \mathrm{mg} \text { injection }\end{array}$ & $\begin{array}{l}12 \\
\text { weeks }\end{array}$ & $\begin{array}{l}\geq 4 \mathrm{~d} / \mathrm{d} \text { for women; } \geq 5 \\
\mathrm{~d} / \mathrm{d} \text { for men }\end{array}$ & OCDS \\
\hline $\begin{array}{l}\text { Pettinati } \\
\text { et al. [18] }\end{array}$ & 2010 & Cognitive behavioral therapy & $100 \mathrm{mg} /$ day & $\begin{array}{l}14 \\
\text { weeks }\end{array}$ & $\begin{array}{l}\geq 4 \mathrm{~d} / \mathrm{d} \text { for women; } \geq 5 \\
\mathrm{~d} / \mathrm{d} \text { for men }\end{array}$ & \\
\hline $\begin{array}{l}\text { Baltieri et } \\
\text { al. [19] }\end{array}$ & 2008 & Relapse prevention counseling & $50 \mathrm{mg} /$ day & $\begin{array}{l}12 \\
\text { weeks }\end{array}$ & & OCDS \\
\hline $\begin{array}{l}\text { Guardia } \\
\text { et al. [20] }\end{array}$ & 2002 & $\begin{array}{l}\text { Supportive group therapy: relapse } \\
\text { prevention + individual counseling }\end{array}$ & $50 \mathrm{mg} /$ day & $\begin{array}{l}12 \\
\text { weeks }\end{array}$ & $\begin{array}{l}>5 \mathrm{~d} / \mathrm{d} \text { for } \mathrm{men} ;>4 \mathrm{~d} / \mathrm{d} \\
\text { for women; or }>5 \mathrm{dd} / \mathrm{w}\end{array}$ & $\begin{array}{l}\text { 11-point } \\
\text { Likert } \\
\text { scale }\end{array}$ \\
\hline $\begin{array}{l}\text { Kiefer et } \\
\text { al. [21] }\end{array}$ & 2003 & $\begin{array}{l}\text { Group therapy: cognitive behavioral } \\
\text { model of substance abuse }\end{array}$ & $50 \mathrm{mg} /$ day & $\begin{array}{l}12 \\
\text { weeks }\end{array}$ & $\begin{array}{l}\geq 5 \mathrm{~d} / \mathrm{d} \text { for men; } \geq 4 \mathrm{~d} / \mathrm{d} \\
\text { for women; or } \geq 5 \mathrm{dd} / \mathrm{w}\end{array}$ & OCDS \\
\hline $\begin{array}{l}\text { Morley et } \\
\text { al. [22] }\end{array}$ & 2006 & No psychotherapy & $50 \mathrm{mg} /$ day & $\begin{array}{l}12 \\
\text { weeks }\end{array}$ & $\begin{array}{l}\geq 4 \mathrm{~d} / \mathrm{d} \text { for women; } \geq 6 \\
d / d \text { for men }\end{array}$ & PACS \\
\hline $\begin{array}{l}\text { Balldin et } \\
\text { al. [23] }\end{array}$ & 2003 & Cognitive behavioral therapy & $50 \mathrm{mg} /$ day & $\begin{array}{l}6 \\
\text { months }\end{array}$ & $\begin{array}{l}\geq 4 \mathrm{~d} / \mathrm{d} \text { for women; } \geq 5 \\
\mathrm{~d} / \mathrm{d} \text { for men }\end{array}$ & OCDS \\
\hline $\begin{array}{l}\text { Balldin et } \\
\text { al. [23] }\end{array}$ & 2003 & Supportive therapy & $50 \mathrm{mg} /$ day & $\begin{array}{l}6 \\
\text { months }\end{array}$ & $\begin{array}{l}\geq 4 \mathrm{~d} / \mathrm{d} \text { for women; } \geq 5 \\
\mathrm{~d} / \mathrm{d} \text { for men }\end{array}$ & OCDS \\
\hline $\begin{array}{l}\text { Chick et } \\
\text { al. [24] }\end{array}$ & 2000 & Psychosocial treatment & $50 \mathrm{mg} /$ day & $\begin{array}{l}12 \\
\text { weeks }\end{array}$ & $\begin{array}{l}\geq 5 \mathrm{~d} / \mathrm{o} \text { for men; } \geq 4 \mathrm{~d} / \mathrm{o} \\
\text { for women }\end{array}$ & \\
\hline $\begin{array}{l}\text { Heinala } \\
\text { et al. [25] }\end{array}$ & 2001 & Coping (group therapy) & $50 \mathrm{mg} /$ day & $\begin{array}{l}12 \\
\text { weeks }\end{array}$ & $\begin{array}{l}\geq 5 \mathrm{~d} / \mathrm{o} \text { or } \geq 5 \mathrm{dd} / \mathrm{w} \text { or } \\
\text { intoxication at site visit }\end{array}$ & \\
\hline $\begin{array}{l}\text { Heinala } \\
\text { et al. [25] }\end{array}$ & 2001 & Supportive therapy & $50 \mathrm{mg} /$ day & $\begin{array}{l}12 \\
\text { weeks }\end{array}$ & $\begin{array}{l}\geq 5 \mathrm{~d} / \mathrm{o} O R \geq 5 \mathrm{dd} / \mathrm{w} \text { or } \\
\text { intoxication at site visit }\end{array}$ & \\
\hline $\begin{array}{l}\text { Ahmadi } \\
\text { et al. [1] }\end{array}$ & 2004 & Counseling & $50 \mathrm{mg} /$ day & $\begin{array}{l}36 \\
\text { weeks }\end{array}$ & $\geq 5 \mathrm{~d} / \mathrm{o}$ or $\geq 5 \mathrm{dd} / \mathrm{w}$ & \\
\hline $\begin{array}{l}\text { Gastpar } \\
\text { et al. [26] }\end{array}$ & 2002 & Psychosocial treatment & $50 \mathrm{mg} /$ day & $\begin{array}{l}12 \\
\text { weeks }\end{array}$ & $\begin{array}{l}\geq 4 \mathrm{~d} / \mathrm{d} \text { for women; } \geq 5 \\
d / d \text { for men }\end{array}$ & \\
\hline $\begin{array}{l}\text { Morris et } \\
\text { al. [27] }\end{array}$ & 2001 & Psychoeducation & $50 \mathrm{mg} /$ day & $\begin{array}{l}12 \\
\text { weeks }\end{array}$ & $\begin{array}{l}\geq 5 \mathrm{~d} / \text { occasion } \text { or } \geq 5 \\
\mathrm{dd} / \mathrm{w} \text { or } \mathrm{BAC}>100 \\
\mathrm{mg} / \mathrm{dl}\end{array}$ & \\
\hline $\begin{array}{l}\text { Latt et al. } \\
\text { [28] }\end{array}$ & 2002 & Counseling and supportive therapy & $50 \mathrm{mg} /$ day & $\begin{array}{l}12 \\
\text { weeks }\end{array}$ & $\geq 5 \mathrm{dd} / \mathrm{w}$ & OCDS \\
\hline $\begin{array}{l}\text { Toneatto } \\
\text { et al. [29] }\end{array}$ & 2009 & Cognitive behavioral therapy & $3 \times 25 \mathrm{mg}+11 \times 50 \mathrm{mg}$ & $\begin{array}{l}12 \\
\text { weeks }\end{array}$ & & \\
\hline $\begin{array}{l}\text { Krystal et } \\
\text { al. [30] }\end{array}$ & 2001 & Counseling & $\begin{array}{l}2 \times 25 \mathrm{mg}+50 \mathrm{mg} / \text { day } \\
\text { thereafter }\end{array}$ & $\begin{array}{l}3 \\
\text { months }\end{array}$ & $\begin{array}{l}\geq 4 \mathrm{~d} / \mathrm{d} \text { for women; } \geq 6 \\
d / d \text { for men }\end{array}$ & \\
\hline
\end{tabular}

\section{TABLE 1: Study characteristics of included studies.}

$d / d=$ drinks per day; $d / o=$ drinks per occasion; $d d / w$ = drinking days per week; BAC = Breath Alcohol Concentration; OCDS = Obsessive Compulsive Drinking scale; AUQ = Alcohol Urge Questionnaire; PACS = Penn Alcohol Craving Scale.

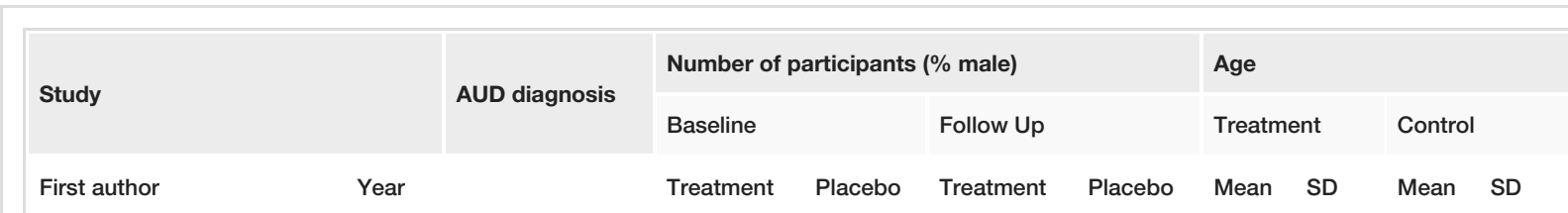




\begin{tabular}{|c|c|c|c|c|c|c|c|c|c|c|}
\hline Anton et al. [4] & 2003 & DSM-III-R AD & $68(69)$ & $63(73)$ & 68 & 63 & 41 & 10 & 44 & 10 \\
\hline Anton et al. [3] (CBI) & $2006 a$ & DSM-IV AD & $155(68)$ & $156(71)$ & 95 & 100 & 45.2 & 10.1 & 43.2 & 9.7 \\
\hline Anton et al. [3] (NP) & $2006 a$ & DSM-IV AD & $154(68)$ & $103(67)$ & 96 & 89 & 44.4 & 9.9 & 44.2 & 9.2 \\
\hline O'Malley et al. [5] (C) & 1992 & DSM-III-R AD & 29 & 25 & 19 & 15 & 42.8 & 10.3 & 38.5 & 8.8 \\
\hline O'Malley et al. [5] (ST) & 1992 & DSM-III-R AD & 23 & 27 & 18 & 16 & & & & \\
\hline Oslin et al. [6] & 1997 & DSM-III-R AD & 21 & 23 & 14 & 13 & 56.5 & 6.8 & 58.9 & 6.7 \\
\hline Petrakis et al. [7] & 2005 & DSM-IV AD & $59(100)$ & $64(100)$ & 46 & 40 & 47.7 & 7.4 & 46.2 & 7.3 \\
\hline Volpicelli et al. [8] & 1997 & DSM-III-R AD & $48(73)$ & $49(82)$ & 35 & 36 & 39 & 9 & 37.9 & 8.5 \\
\hline Davidson et al. [9] & 2004 & AUDIT $\geq 8$ & 19 & 19 & 16 & 19 & 46.5 & 10.5 & 50.8 & 7 \\
\hline Kranzler et al. [10] & 2000 & DSMIII-R AD & $61(80)$ & $63(75)$ & & & 39.7 & 8.4 & 41.8 & 8.1 \\
\hline Monti et al. [11] & 2001 & DSM-IV AD/A & 64 & 64 & & & & & & \\
\hline O'Malley et al. [12] (NP) & 2003 & DSM-III-R AD & 26 & 27 & 17 & 13 & & & & \\
\hline O'Malley et al. [12] (CBT) & 2003 & DSM-III-R AD & 30 & 30 & 19 & 24 & & & & \\
\hline O'Malley et al. [13] & 2008 & DSM-IV AD & $34(65)$ & $34(62)$ & 35 & 21 & 42 & 10.67 & 38.8 & 10.41 \\
\hline Oslin [14] & 2005 & DSM-IV AD & $37(78)$ & $37(81)$ & & & 64.2 & 6.9 & 62.5 & 5.6 \\
\hline Pettinati et al. [15] & 2006 & DSM-IV AD & $52(75)$ & $54(70)$ & 35 & 32 & 41.3 & 6.8 & 41.2 & 7.5 \\
\hline Brown et al. [16] & 2009 & DSM-IV AD & $20(50)$ & $20(45)$ & 14 & 12 & & & & \\
\hline Anton et al. [2] (CBT) & 2005 & DSM-IV AD & $39(79)$ & $41(73)$ & 36 & 37 & 44 & 8 & 45 & 11 \\
\hline Anton et al. [2] (MET) & 2005 & DSM-IV AD & $41(73)$ & $39(77)$ & 34 & 28 & 43 & 10 & 43 & 9 \\
\hline Kranzler et al. [17] & 2004 & DSM-IV AD & $158(67)$ & $157(63)$ & 127 & 118 & 44.1 & 9.6 & 43.6 & 8.5 \\
\hline Pettinati et al. [18] & 2010 & DSM-IV AD & $49(67)$ & $39(56)$ & 29 & 23 & 42.9 & 8.1 & 43.4 & 8.9 \\
\hline Baltieri et al. [19] & 2008 & ICD-10 AD & $49(100)$ & $54(100)$ & 29 & 23 & 44.1 & 7.2 & 43.4 & 8.8 \\
\hline Guardia et al. [20] & 2002 & DSM-IV AD & $101(72)$ & $101(77)$ & 61 & 59 & 41 & 8 & 42 & 9 \\
\hline Kiefer et al. [21] & 2003 & DSM-IV AD & $40(78)$ & $40(68)$ & 22 & 10 & 46.1 & 8.1 & 45.6 & 11.1 \\
\hline Morley et al. [22] & 2006 & DSM-IV AD/A & $53(72)$ & $61(64)$ & 36 & 40 & 47.6 & 8.5 & 42.4 & 9.3 \\
\hline Balldin et al. [23] (CBT) & 2003 & DSM-IV AD & $25(84)$ & $30(77)$ & & & 50 & 7 & 50 & 8 \\
\hline Balldin et al. [23] (ST) & 2003 & DSM-IV AD & $31(87)$ & $32(91)$ & & & 48 & 8 & 51 & 8 \\
\hline Chick et al. [24] & 2000 & DSM-III-R AD/A & $90(72)$ & $85(78)$ & 37 & 36 & 43.1 & 8.3 & 43.9 & 9.7 \\
\hline Heinala et al. [25] (C) & 2001 & DSM-IV AD & 34 & 33 & & & & & & \\
\hline Heinala et al. [25] (ST) & 2001 & DSM-IV AD & 29 & 25 & & & & & & \\
\hline Ahmadi et al. [1] & 2004 & DSM-IV AD & 58 & 58 & 26 & 15 & 42.76 & 9.58 & 43.19 & 8.81 \\
\hline Gastpar et al. [26] & 2002 & DSM-III-R AD/A & $84(77)$ & $87(68)$ & 56 & 54 & 43.4 & 9.9 & 42 & 9.6 \\
\hline Morris et al. [27] & 2001 & DSM-III-R AD & $55(100)$ & $56(100)$ & & & 47 & 8 & 48 & 8 \\
\hline Latt et al. [28] & 2002 & DSM-IV AD & 56 & 51 & 38 & 36 & & & & \\
\hline Toneatto et al. [29] & 2009 & DSM-IV AD/A & 27 & 25 & 26 & 25 & & & & \\
\hline Krystal et al. [30] & 2001 & DSM-IV AD & $418(97)$ & $209(98)$ & 378 & 187 & 48.9 & 10 & 49.5 & 10 \\
\hline
\end{tabular}

\section{TABLE 2: Participant characteristics of included studies.}

AUD = Alcohol use disorders; DSM = Diagnostic and Statistic Manual; SD = Standard Deviation; C = Coping; CBT = Cognitive Behavioral Therapy; ST = Supportive Therapy; NP = No psychotherapy; AD = Alcohol Dependence; AD/A = Alcohol dependence or abuse; AUDIT = Alcohol Use Disorder Inventory Test; $\mathrm{MET}=$ Motivational Enhancement Therapy; $\mathrm{CBI}=$ Combined Behavioral Intervention. 


\section{Cureus}

\begin{tabular}{|c|c|c|c|c|c|c|c|}
\hline Model/Parameter & $\mathbf{K}$ & $\mathrm{N}_{\text {treatment }}$ & $\mathbf{N}_{\text {control }}$ & Estimate & $95 \% \mathrm{Cl}$ & $\mathbf{Q}$ & $P_{\text {with vs. w/o psychotherapy }}$ \\
\hline \multicolumn{8}{|l|}{ Abstinence } \\
\hline Random Effect Model (REM) & 17 & 954 & 959 & $1.36^{*}$ & $1.06,1.75$ & 25.26 & \\
\hline With psychotherapy & 13 & 771 & 763 & $1.45^{\star}$ & $1.10,1.91$ & 22.01 & \multirow{2}{*}{.43} \\
\hline w/o psychotherapy & 4 & 183 & 196 & 1.10 & $.59,2.06$ & 5.69 & \\
\hline Mixed Effect Model (MEM) & 17 & 954 & 959 & & & 27.70 & \\
\hline Intercept & & & & .07 & $-.47, .61$ & & \\
\hline Psychotherapy & & & & .27 & $-.34, .88$ & & \\
\hline \multicolumn{8}{|l|}{ Relapse } \\
\hline REM & 27 & 1990 & 1725 & $.66^{\star \star \star}$ & $.57, .77$ & $40.33^{*}$ & \\
\hline With psychotherapy & 23 & 1656 & 1434 & $.65^{\star \star \star}$ & $.55, .77$ & $35.59^{*}$ & \multirow{3}{*}{.67} \\
\hline w/o psychotherapy & 4 & 334 & 286 & $.70^{\star}$ & $-.68, .98$ & 4.59 & \\
\hline MEM & 27 & 1990 & 1725 & & & $41.80^{*}$ & \\
\hline Intercept & & & & $-.35^{\star}$ & $-.69-.02$ & & \\
\hline Psychotherapy & & & & -.07 & $-.44, .30$ & & \\
\hline \multicolumn{8}{|c|}{ Gamma Glutamyl Transferase (GGT) } \\
\hline REM & 13 & 554 & 565 & $-.16^{\star}$ & $-.29,-.04$ & 3.74 & \\
\hline With psychotherapy & 9 & 379 & 389 & -.15 & $-.30, .01$ & 2.37 & \multirow{2}{*}{.74} \\
\hline w/o psychotherapy & 4 & 175 & 176 & -.19 & $-.41, .03$ & 1.26 & \\
\hline MEM & 13 & 554 & 565 & & & 3.29 & \\
\hline Intercept & & & & -.19 & $-.41, .03$ & & \\
\hline Psychotherapy & & & & -.05 & $-.22, .32$ & & \\
\hline \multicolumn{8}{|l|}{ Cravings } \\
\hline REM & 14 & 626 & 640 & -.11 & $-.31, .10$ & 2.36 & \\
\hline With psychotherapy & 10 & 457 & 467 & -.19 & $-.42, .03$ & 15.55 & \multirow{3}{*}{.14} \\
\hline w/o psychotherapy & 4 & 169 & 173 & .14 & $-.24, .51$ & 4.42 & \\
\hline MEM & 14 & 626 & 640 & & & $23.38^{\star}$ & \\
\hline Intercept & & & & .14 & $-.23, .51$ & & \\
\hline Psychotherapy & & & & -.33 & $-.76, .10$ & & \\
\hline
\end{tabular}

TABLE 3: Results of the meta-analyses.

Note: Estimates for REM in the abstinence and relapse models are presented as odds ratios.

$\mathrm{K}=$ Number of studies; $\mathrm{Cl}$ = Confidence intervals; w/o = without; GGT = Gamma-Glutamyl Transferase; REM = Random Effects Model; MEM = Mixed Effects Model.

${ }^{*} \mathrm{p}<.05 ;{ }^{* *} \mathrm{p}<.01 ;{ }^{* * *} \mathrm{p}<.001$ 


\section{Cureus}

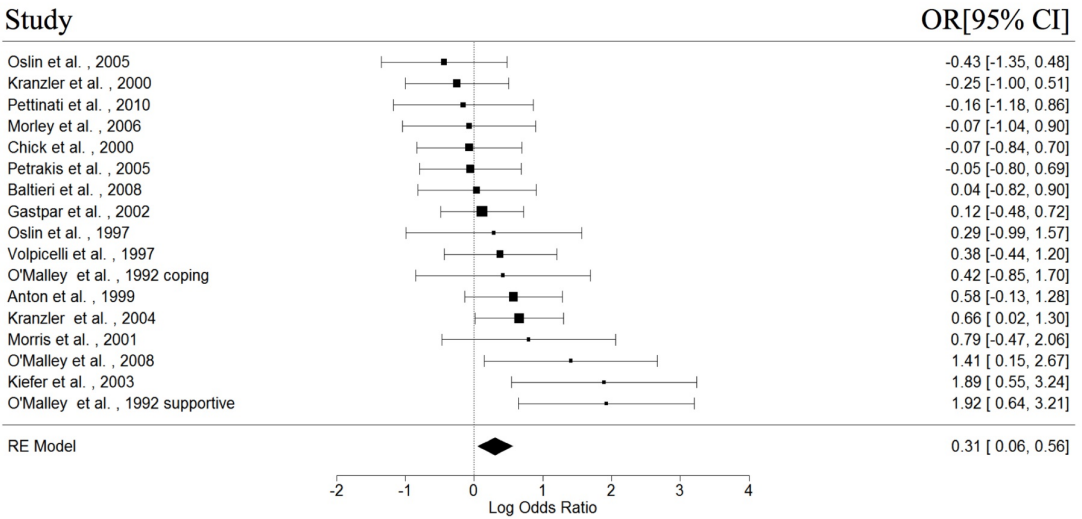

FIGURE 2: Forest plots of effect sizes for abstinence.

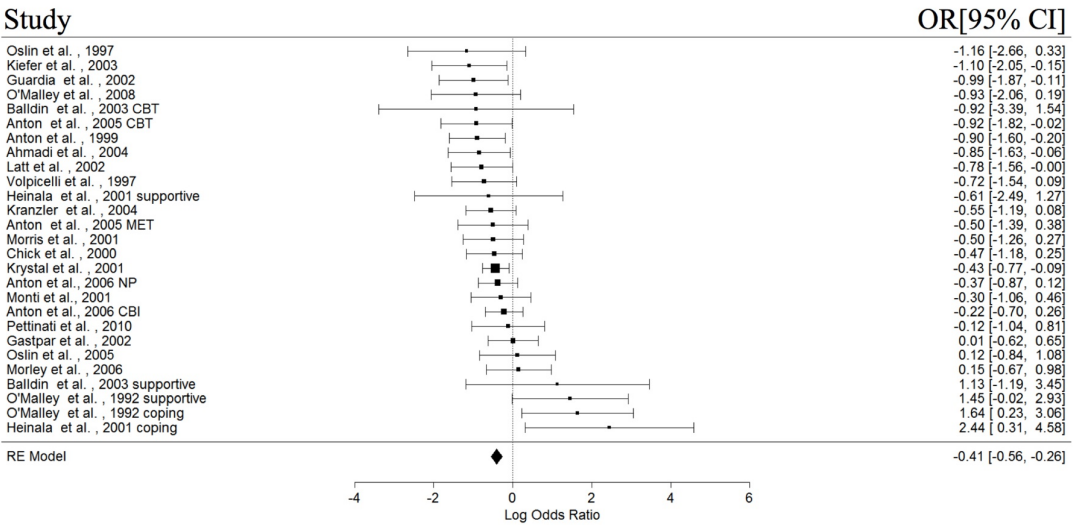

FIGURE 3: Forest plots of effect sizes for relapse.

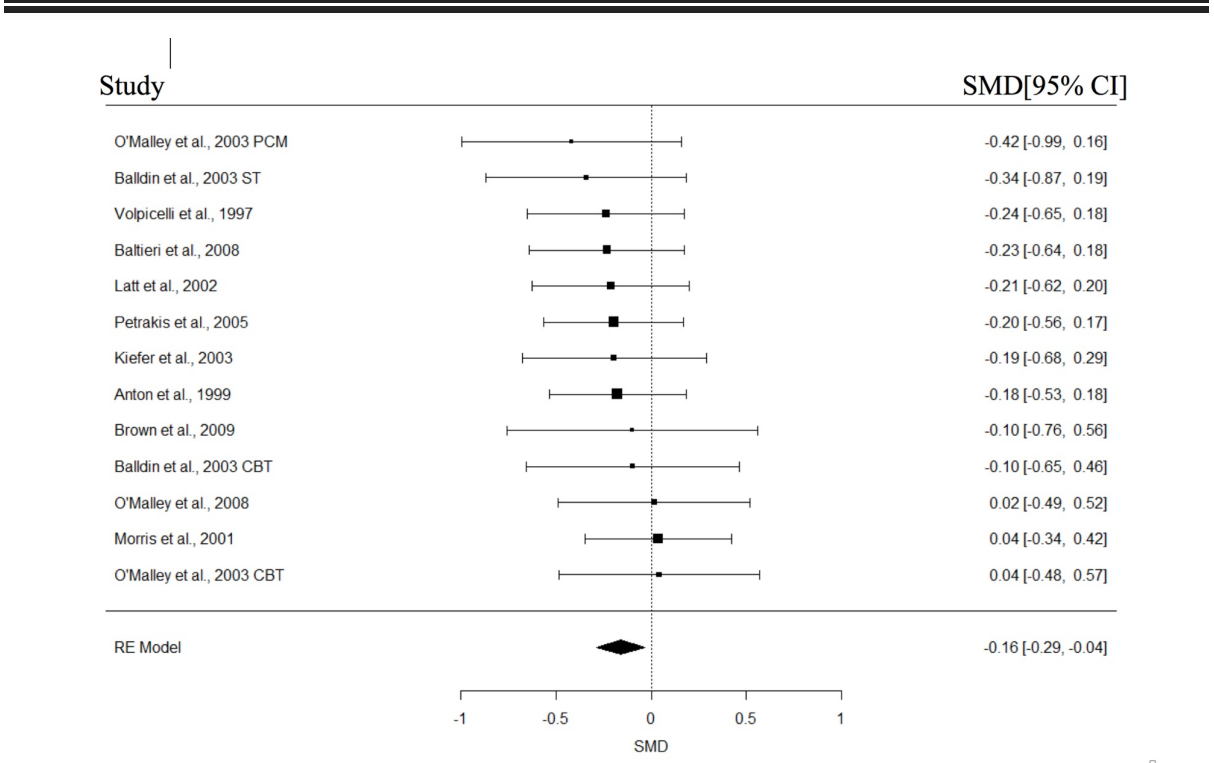

FIGURE 4: Forest plots of effect sizes for Gamma-Glutamyl Transferase. 


\section{Cureus}

\begin{tabular}{l} 
Brown et al., 2009 \\
Kranzler et al., 2000 \\
Balldin et al, 2003 CBT \\
O'Malley et al, 2008 \\
Anton et al., 1999 \\
Balldin et al, 2003 ST \\
Baltieri et al., 2008 \\
Kiefer et al, 2003 \\
O'Malley et al, 2003 PCM \\
Guardia et al, 2002 \\
O'Malley et al., 2003 CBT \\
Latt et al., 2002 \\
Morley et al., 2006 \\
Davison et al, 2004 \\
\hline RE Model
\end{tabular}

FIGURE 5: Forest plots of effect sizes for cravings.

The Q statistics indicated that there was significant heterogeneity in the REMs of all studies and studies with psychotherapy for relapse. Furthermore, there was also significant heterogeneity in the MEMs for relapse, and cravings. The trim and fill analyses carried out for all REMs of all studies did not result in any imputation of studies. This suggests that publication biases were minimal or non-significant. The funnel plots and leave-one-out analyses are presented in Figures 6-9 and Tables 4-7. In general, the exclusion of any single study from the REMs did not alter the statistical significance of any existing pooled estimates; these estimates remained statistically significant in the REMs for abstinence, relapse, and GGT, and nonsignificant in the REM for cravings. These results suggest that the results are generally robust.

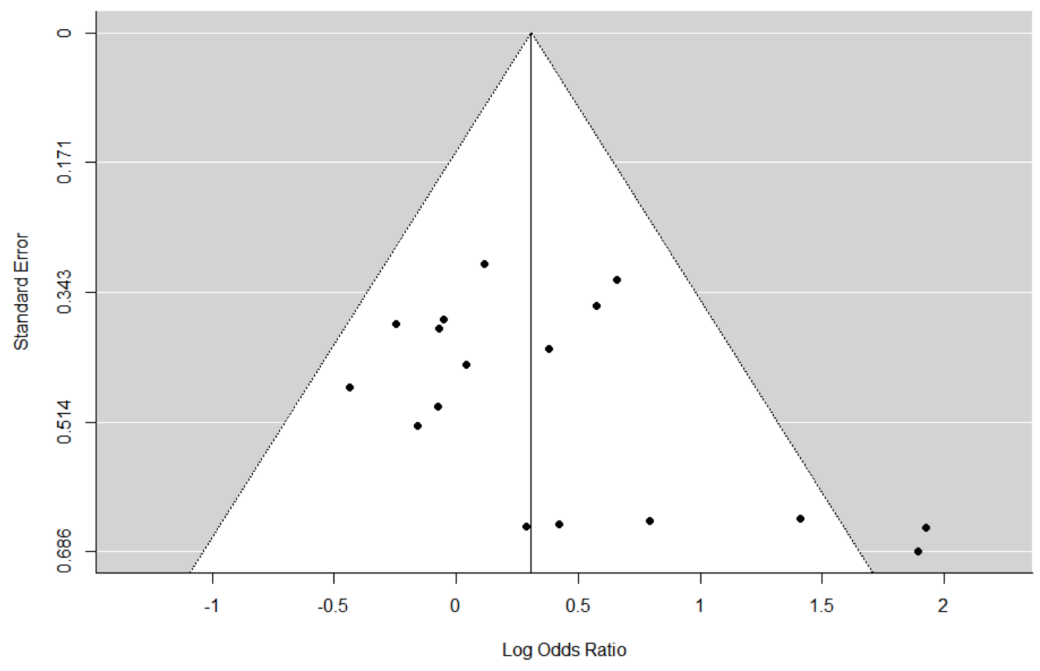

FIGURE 6: Funnel plots of standard errors plotted against effect sizes for identification of publication bias for abstinence. 


\section{Cureus}

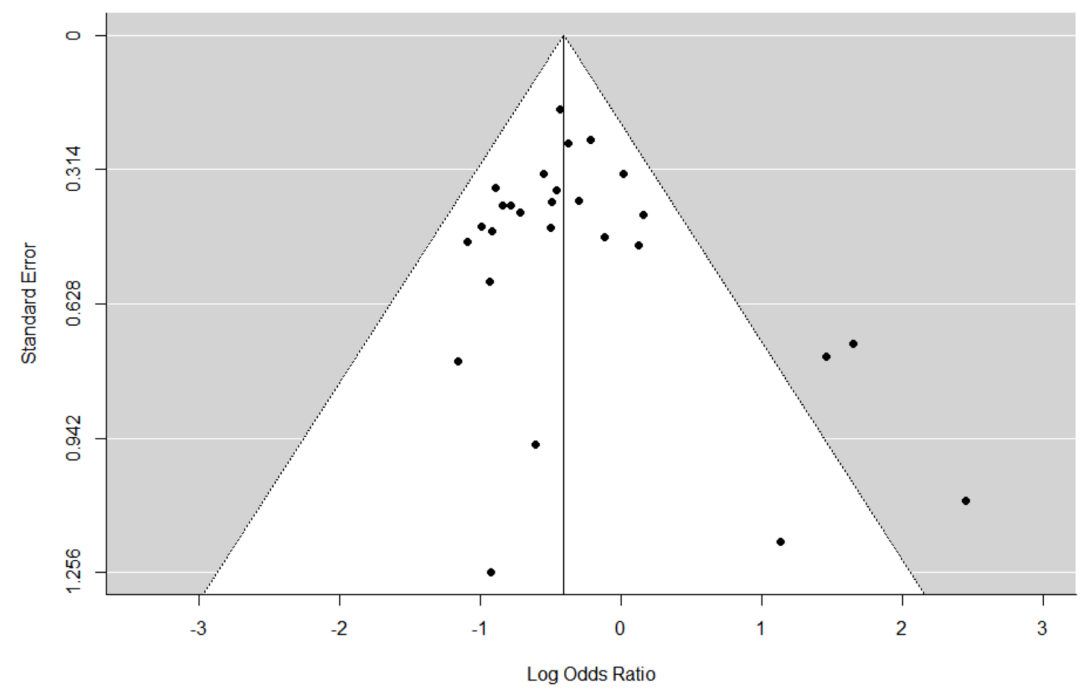

FIGURE 7: Funnel plots of standard errors plotted against effect sizes for identification of publication bias for relapse.

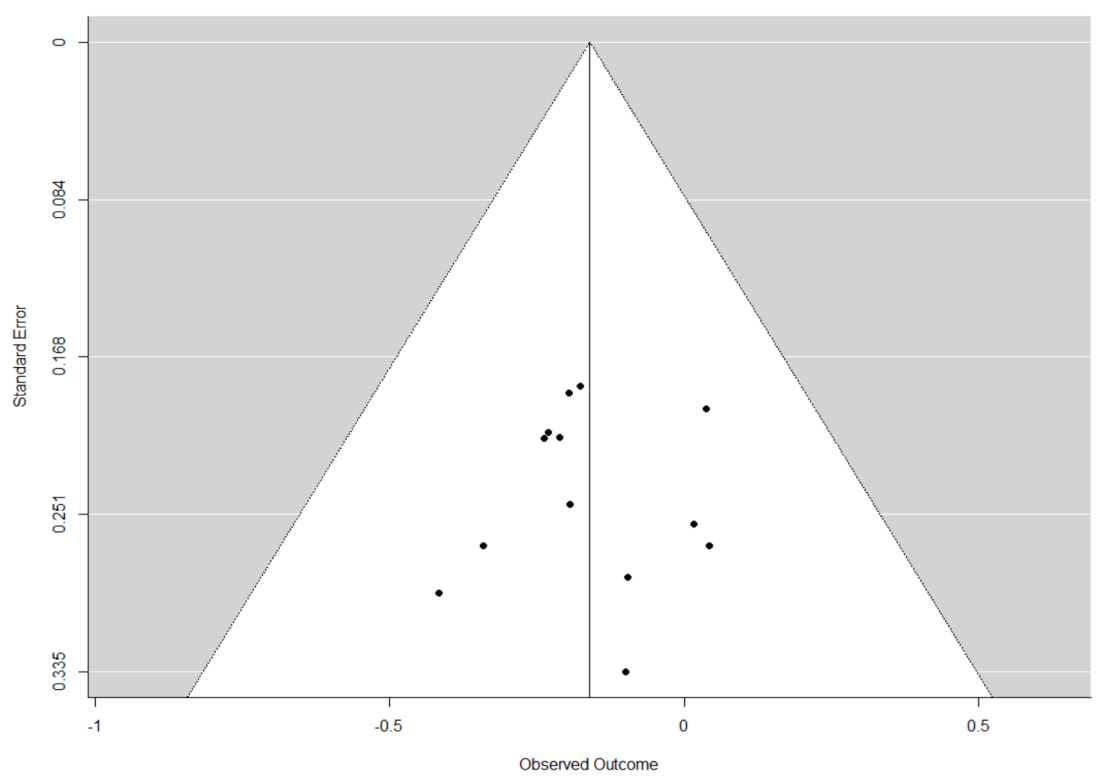

FIGURE 8: Funnel plots of standard errors plotted against effect sizes for identification of publication bias for Gamma-Glutamyl Transferase. 


\section{Cureus}

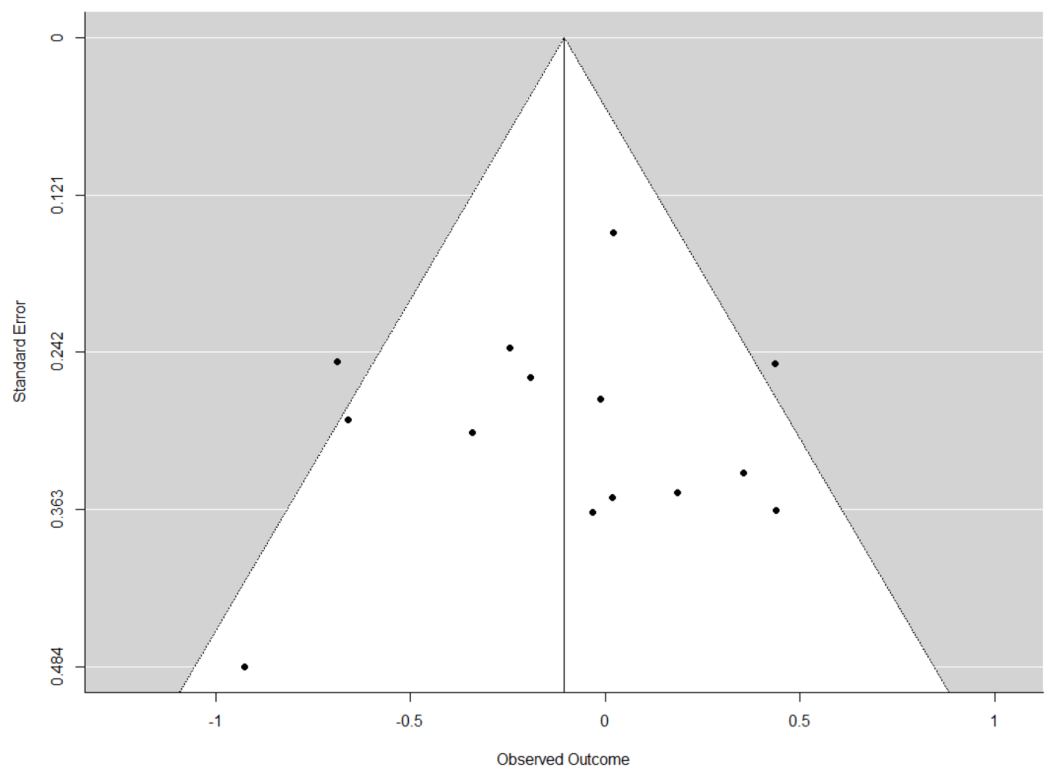

FIGURE 9: Funnel plots of standard errors plotted against effect sizes for identification of publication bias for cravings.

\begin{tabular}{|c|c|c|c|c|c|c|c|}
\hline \multirow[b]{2}{*}{1} & \multirow{2}{*}{$\begin{array}{l}\text { Study/Year } \\
\text { O'Malley et al. [5] (C)(1992) }\end{array}$} & \multirow{2}{*}{$\begin{array}{l}\text { Estimate } \\
0.31\end{array}$} & \multirow{2}{*}{$\begin{array}{l}P_{\text {Estimate }} \\
0.0227\end{array}$} & \multicolumn{2}{|c|}{$95 \% \mathrm{Cl}$} & \multirow{2}{*}{$\begin{array}{l}Q \\
25.22\end{array}$} & \multirow{2}{*}{$\begin{array}{l}P_{Q} \\
0.0471\end{array}$} \\
\hline & & & & 0.04 & 0.57 & & \\
\hline 2 & O'Malley et al. [5] (ST) (1992) & 0.24 & 0.0331 & 0.02 & 0.46 & 18.82 & 0.2220 \\
\hline 3 & Oslin et al. [6] (1997) & 0.31 & 0.0209 & 0.05 & 0.58 & 25.26 & 0.0465 \\
\hline 4 & Volpicelli et al. [8] (1997) & 0.31 & 0.0280 & 0.03 & 0.59 & 25.21 & 0.0472 \\
\hline 5 & Anton et al. [4] (2003) & 0.29 & 0.0380 & 0.02 & 0.56 & 24.56 & 0.0562 \\
\hline 6 & Kranzler et al. [10] (2000) & 0.35 & 0.0076 & 0.09 & 0.61 & 23.18 & 0.0803 \\
\hline 7 & Chick et al. [24] (2000) & 0.34 & 0.0134 & 0.07 & 0.62 & 24.37 & 0.0591 \\
\hline 8 & Morris et al. [27] (2001) & 0.29 & 0.0275 & 0.03 & 0.55 & 24.62 & 0.0552 \\
\hline 9 & Gastpar et al. [26] (2002) & 0.34 & 0.0201 & 0.05 & 0.62 & 24.92 & 0.0511 \\
\hline 10 & Kiefer et al. [21] (2003) & 0.25 & 0.0326 & 0.02 & 0.48 & 19.63 & 0.1867 \\
\hline 11 & Kranzler et al. [17] (2004) & 0.27 & 0.0449 & 0.01 & 0.53 & 23.81 & 0.0683 \\
\hline 12 & Petrakis et al. [7] (2005) & 0.34 & 0.0137 & 0.07 & 0.62 & 24.39 & 0.0588 \\
\hline 13 & Oslin [14] (2005) & 0.35 & 0.0068 & 0.10 & 0.6 & 22.76 & 0.0893 \\
\hline 14 & Morley et al. [22] (2006) & 0.33 & 0.0149 & 0.07 & 0.6 & 24.71 & 0.0539 \\
\hline 15 & O'Malley et al. [13] (2008) & 0.26 & 0.0325 & 0.02 & 0.5 & 22.11 & 0.1048 \\
\hline 16 & Baltieri et al. [19] (2008) & 0.33 & 0.0173 & 0.06 & 0.61 & 24.93 & 0.0509 \\
\hline 17 & Pettinati et al. [18] (2010) & 0.34 & 0.0135 & 0.07 & 0.6 & 24.51 & 0.0570 \\
\hline
\end{tabular}

\section{TABLE 4: Leave-one-out sensitivity analysis for abstinence.}

C = Coping; ST = Supportive Therapy. 


\begin{tabular}{|c|c|c|c|c|c|c|c|}
\hline \multirow[b]{2}{*}{1} & \multirow{2}{*}{$\begin{array}{l}\text { Study } \\
\text { O'Malley et al. [5] (C)(1992) }\end{array}$} & \multirow{2}{*}{$\begin{array}{l}\text { Estimate } \\
-0.44\end{array}$} & \multirow{2}{*}{$\begin{array}{l}\text { PEstimate } \\
<.0001>\end{array}$} & \multicolumn{2}{|c|}{$95 \% \mathrm{Cl}$} & \multirow{2}{*}{$\begin{array}{l}\text { Q } \\
33.74\end{array}$} & \multirow{2}{*}{$\begin{array}{l}P_{Q} \\
0.1417\end{array}$} \\
\hline & & & & -0.59 & -0.29 & & \\
\hline 2 & O'Malley et al. [5] (ST) (1992) & -0.44 & $<.0001>$ & -0.58 & -0.29 & 35.76 & 0.0961 \\
\hline 3 & Anton et al. [3] (CBI) (2006) & -0.44 & $<.0001>$ & -0.59 & -0.28 & 41.27 & 0.0292 \\
\hline 4 & Anton et al. [3] (NP) (2006) & -0.42 & $<.0001>$ & -0.57 & -0.27 & 41.95 & 0.0248 \\
\hline 5 & Oslin et al. [6] (1997) & -0.41 & $<.0001>$ & -0.56 & -0.26 & 41.01 & 0.0310 \\
\hline 6 & Volpicelli et al. [8] (1997) & -0.41 & $<.0001>$ & -0.56 & -0.26 & 41.42 & 0.0282 \\
\hline 7 & Anton et al. [4] (2003) & -0.39 & $<.0001>$ & -0.54 & -0.24 & 40.09 & 0.0382 \\
\hline 8 & Chick et al. [24] (2000) & -0.41 & $<.0001>$ & -0.56 & -0.26 & 41.96 & 0.0248 \\
\hline 9 & Krystal et al. [30] (2001) & -0.41 & $<.0001>$ & -0.58 & -0.25 & 41.97 & 0.0247 \\
\hline 10 & Heinala et al. [25] (C)(2001) & -0.43 & $<.0001>$ & -0.58 & -0.28 & 35.02 & 0.1111 \\
\hline 11 & Heinala et al. [25] (ST) (2001) & -0.42 & $<.0001>$ & -0.56 & -0.27 & 41.94 & 0.0249 \\
\hline 12 & Morris et al. [27] (2001) & -0.41 & $<.0001>$ & -0.56 & -0.26 & 41.94 & 0.0249 \\
\hline 13 & Guardia et al. [20] (2002) & -0.40 & $<.0001>$ & -0.55 & -0.25 & 40.29 & 0.0366 \\
\hline 14 & Gastpar et al. [26] (2002) & -0.44 & $<.0001>$ & -0.59 & -0.29 & 40.13 & 0.0379 \\
\hline 15 & Latt et al. [28] (2002) & -0.40 & $<.0001>$ & -0.55 & -0.25 & 41.09 & 0.0304 \\
\hline 16 & Kiefer et al. [21] (2003) & -0.40 & $<.0001>$ & -0.55 & -0.25 & 39.93 & 0.0396 \\
\hline 17 & Balldin et al. [23] (2003) (CBT) & -0.41 & $<.0001>$ & -0.56 & -0.27 & 41.81 & 0.0257 \\
\hline 18 & Balldin et al. [23] (2003) (ST) & -0.42 & $<.0001>$ & -0.57 & -0.28 & 40.26 & 0.0368 \\
\hline 19 & Kranzler et al. [17] (2004) & -0.41 & $<.0001>$ & -0.56 & -0.26 & 41.79 & 0.0258 \\
\hline 20 & Ahmadi et al. [1] (2004) & -0.40 & $<.0001>$ & -0.55 & -0.25 & 40.78 & 0.0326 \\
\hline 21 & Oslin [14] (2005) & -0.43 & $<.0001>$ & -0.58 & -0.28 & 40.76 & 0.0328 \\
\hline 22 & Anton et al. [2] (2005) (CBT) & -0.40 & $<.0001>$ & -0.55 & -0.25 & 40.76 & 0.0328 \\
\hline 23 & Anton et al. [2] (2005) (MET) & -0.41 & $<.0001>$ & -0.56 & -0.26 & 41.94 & 0.0249 \\
\hline 24 & Morley et al. [22] (2006) & -0.44 & $<.0001>$ & -0.59 & -0.29 & 40.07 & 0.0384 \\
\hline 25 & O'Malley et al. [13] (2008) & -0.41 & $<.0001>$ & -0.56 & -0.26 & 41.15 & 0.0299 \\
\hline 26 & Toneatto et al. [29] (2009) & -0.41 & $<.0001>$ & -0.56 & -0.26 & 40.33 & 0.0362 \\
\hline 27 & Pettinati et al. [18] (2010) & -0.42 & $<.0001>$ & -0.57 & -0.28 & 41.56 & 0.0272 \\
\hline 28 & Monti et al. [11] (2001) & -0.42 & $<.0001>$ & -0.57 & -0.27 & 41.89 & 0.0252 \\
\hline
\end{tabular}

\section{TABLE 5: Leave-one-out sensitivity analysis for relapse.}

C = Coping; ST = Supportive Therapy; CBI = Combined Behavioral Intervention; NP = No psychotherapy; CBT = Cognitive Behavioral Therapy; MET = Motivational Enhancement Therapy. 


\section{Cureus}

\begin{tabular}{|c|c|c|c|c|c|c|c|}
\hline \multirow[b]{2}{*}{1} & \multirow{2}{*}{$\begin{array}{l}\text { Study/Year } \\
\text { Anton et al. [4] (2003) }\end{array}$} & \multirow{2}{*}{$\begin{array}{l}\text { Estimate } \\
-0.16\end{array}$} & \multirow{2}{*}{$\begin{array}{l}P_{\text {Estimate }} \\
0.0203\end{array}$} & \multicolumn{2}{|c|}{$95 \% \mathrm{Cl}$} & \multirow{2}{*}{$\begin{array}{l}\text { Q } \\
3.74\end{array}$} & \multirow{2}{*}{$\begin{array}{l}P_{Q} \\
0.9769\end{array}$} \\
\hline & & & & -0.29 & -0.02 & & \\
\hline 2 & Petrakis et al. [7] (2005) & -0.16 & 0.0221 & -0.29 & -0.02 & 3.71 & 0.9777 \\
\hline 3 & Volpicelli et al. [8] (1997) & -0.15 & 0.0229 & -0.28 & -0.02 & 3.60 & 0.9802 \\
\hline 4 & O'Malley et al. [12] (2003) (PCM) & -0.15 & 0.0243 & -0.28 & -0.02 & 2.94 & 0.9915 \\
\hline 5 & O'Malley et al. [12] (2003) (CBT) & -0.17 & 0.0088 & -0.30 & -0.04 & 3.14 & 0.9887 \\
\hline 6 & O'Malley et al. [13] (2008) & -0.17 & 0.0091 & -0.30 & -0.04 & 3.24 & 0.9871 \\
\hline 7 & Brown et al. [16] (2009) & -0.16 & 0.0125 & -0.29 & -0.03 & 3.71 & 0.9776 \\
\hline 8 & Baltieri et al. [19] (2008) & -0.15 & 0.0228 & -0.28 & -0.02 & 3.62 & 0.9798 \\
\hline 9 & Kiefer et al. [21] (2003) & -0.16 & 0.0170 & -0.29 & -0.03 & 3.73 & 0.9772 \\
\hline 10 & Balldin et al. [23] (2003) (CBT) & -0.16 & 0.0125 & -0.29 & -0.04 & 3.69 & 0.9781 \\
\hline 11 & Balldin et al. [23] (2003) (ST) & -0.15 & 0.0231 & -0.28 & -0.02 & 3.27 & 0.9867 \\
\hline 12 & Morris et al. [27] (2001) & -0.18 & 0.0065 & -0.32 & -0.05 & 2.59 & 0.9951 \\
\hline 13 & Latt et al. [28] (2002) & -0.15 & 0.0207 & -0.29 & -0.02 & 3.68 & 0.9783 \\
\hline
\end{tabular}

TABLE 6: Leave-one-out sensitivity analysis for Gamma-Glutamyl Transferase.

$\mathrm{PCM}=$ Primary Care Management; $\mathrm{CBT}$ = Cognitive Behavioral Therapy.

\begin{tabular}{|c|c|c|c|c|c|c|c|}
\hline \multirow[b]{2}{*}{1} & \multirow{2}{*}{$\begin{array}{l}\text { Study/Year } \\
\text { Anton et al. [4] (2003) }\end{array}$} & \multirow{2}{*}{$\begin{array}{l}\text { Estimate } \\
-0.09\end{array}$} & \multirow{2}{*}{$\begin{array}{l}P_{\text {Estimate }} \\
0.4124\end{array}$} & \multicolumn{2}{|c|}{$95 \% \mathrm{Cl}$} & \multirow{2}{*}{$\begin{array}{l}\mathbf{Q} \\
22.94\end{array}$} & \multirow{2}{*}{$\begin{array}{l}P_{Q} \\
0.0282\end{array}$} \\
\hline & & & & -0.31 & 0.13 & & \\
\hline 2 & Davison et al. [9] (2004) & -0.14 & 0.1903 & -0.34 & 0.07 & 21.14 & 0.0484 \\
\hline 3 & Kranzler et al. [10] (2000) & -0.05 & 0.6108 & -0.23 & 0.13 & 17.18 & 0.1430 \\
\hline 4 & O'Malley et al. [12] (2003) (PCM) & -0.11 & 0.3018 & -0.33 & 0.10 & 23.28 & 0.0254 \\
\hline 5 & O'Malley et al. [12] (2003) (CBT) & -0.12 & 0.2539 & -0.34 & 0.09 & 22.72 & 0.0302 \\
\hline 6 & O'Malley et al. [13] (2008) & -0.09 & 0.4202 & -0.30 & 0.13 & 22.70 & 0.0304 \\
\hline 7 & Brown et al. [16] (2009) & -0.08 & 0.4584 & -0.28 & 0.12 & 20.37 & 0.0603 \\
\hline 8 & Baltieri et al. [19] (2008) & -0.11 & 0.3152 & -0.32 & 0.10 & 23.36 & 0.0249 \\
\hline 9 & Guardia et al. [20] (2002) & -0.12 & 0.2898 & -0.35 & 0.10 & 22.61 & 0.0312 \\
\hline 10 & Kiefer et al. [21] (2003) & -0.11 & 0.3106 & -0.33 & 0.11 & 23.29 & 0.0253 \\
\hline 11 & Morley et al. [22] (2006) & -0.15 & 0.1115 & -0.35 & 0.04 & 18.49 & 0.1016 \\
\hline 12 & Balldin et al. [23] (2003) (CBT) & -0.06 & 0.5330 & -0.26 & 0.13 & 19.46 & 0.0779 \\
\hline 13 & Balldin et al. [23] (2003) (ST) & -0.10 & 0.3821 & -0.32 & 0.12 & 23.24 & 0.0258 \\
\hline 14 & Latt et al. [28] (2002) & -0.13 & 0.1999 & -0.34 & 0.07 & 21.49 & 0.0437 \\
\hline
\end{tabular}

\section{Discussion}


The current study examined the hypothesis that naltrexone is efficacious in treating AUD and psychotherapy on top of naltrexone significantly augments AUD-related treatment outcomes. In relation to former, our results have generally indicated that naltrexone has been efficacious in treating AUD. Specifically, the pool estimates indicated significant treatment effects in improving self-reported alcohol consumption outcomes such as abstinence, relapse as well as the objectively measured GGT, which is a widely used and highly specific biomarker for excessive alcohol consumption. However, there was not a significant treatment effect on cravings. In relation to the second hypothesis, adding psychotherapy to the naltrexone treatment of AUD did not significantly augment treatment outcomes; the combined treatment of psychotherapy and naltrexone was not significantly better than naltrexone alone.

The non-significant difference in treatment outcomes between studies with and without psychotherapy is noteworthy considering that previous meta-analytic research had found combined treatments significantly more effective than pharmacological treatment alone in treating mood and anxiety disorders. Nevertheless, while previous meta-analyses have noted significant and large treatment effects associated with psychotherapy among these affective disorders, psychotherapy has been less successful with AUDs. Metaanalyses have generally reported small and non-significant treatment effects associated with psychotherapy on AUD. Given these weak effects, one would not expect the addition of psychotherapy to significantly augment the naltrexone treatment of AUD. Indeed, researchers who investigated such a hypothesis withinstudy did not find significant differences between combined treatment and naltrexone-only treatment in influencing subsequent AUD outcomes [2, 3]. One may be concerned at the large variety of psychotherapies carried out across studies, thus rendering it difficult to interpret or generalize these results to the different adjunctive psychotherapies. This may be the case for the meta-analyzed relapse outcome, which was associated with significant heterogeneity. Notwithstanding the slight variation in the definition of relapse across studies, it may be possible that certain therapies when combined with naltrexone yield much better treatment outcomes than others. Nevertheless, despite the wide variety of psychotherapies carried out across the different studies, the results among studies with psychotherapy were not significantly heterogeneous, at least in relation to abstinence, GGT and cravings. Assuming the naltrexone effect is constant across study, this may hint to the dodo bird verdict - all psychotherapies, regardless of their theoretical orientations, produced similar outcomes. Regardless, future researchers may consider comparing treatment outcomes between various psychotherapies in combination with naltrexone to verify such an interpretation.

It is also interesting that while naltrexone had resulted in significantly improved treatment outcomes in terms of self-reported measures and biomarkers of alcohol consumption, it did not seem to have a significant treatment effect in reducing alcohol-related cravings. There may be two explanations for this. First, while naltrexone reportedly modulates the dopaminergic activity in the mid-brain reward system in an attempt to inhibit the reinforcement associated with alcohol consumption, it does little to alleviate alcoholrelated cravings which are largely associated with activity in the prefrontal and limbic regions of the brain. Second, it is plausible that alcohol-related cravings will decrease on its own even without any naltrexone treatment and this decrease will therefore mask the naltrexone treatment effect, if any at all on cravings. Indeed, we observed that several studies reported significant decreases (with respect to baseline) in cravings among placebo groups which were comparable to those of the naltrexone treatment groups. Furthermore, this decrease cannot be explained by the adjunctive psychotherapy in most of the included studies since it was not a significant moderator in the MEM; the pooled estimates relating to the decrease in cravings were also similar between studies with and without psychotherapy.

These findings present a key implication in the treatment of AUD. These results suggest that it is not necessary for adjunctive psychotherapy to be carried out on top of naltrexone in the treatment of AUD. From a resource-allocation perspective, given that individual psychotherapy is a relatively time- and resource-consuming process, the resources associated with such adjunctive psychotherapies can be conserved, or instead should be directed at other clinical populations, such as those with depression and anxiety disorders, in which psychotherapy would be a lot more beneficial.

The current results are subjected to three major limitations. Firstly, given the very limited number of studies on naltrexone treatment without psychotherapy, their pooled estimates may not reliably reflect the effect of naltrexone treatment alone. More 'naltrexone-only' intervention studies should be carried out such that future meta-analyses can robustly compare the AUD treatment outcomes between combined-intervention and naltrexone-only treatments. Secondly, it is possible that between-study differences in methodology or participants' characteristics among the included studies may confound the inclusion of psychotherapy in influencing treatment outcomes. Future intervention studies on AUD should be carried out to examine the inclusion of psychotherapy on top of naltrexone treatment within-study, such that stronger conclusions relating to the addition of psychotherapy on top of naltrexone can be made. Thirdly, the manner in which the additive effects of psychotherapy were studied in the current meta-analysis was less than optimal, given that we have simply compared studies with or without psychotherapy, instead of studies with psychotherapy or a control condition for psychotherapy. Furthermore, unlike the naltrexone condition, the psychotherapy conditions were not randomly assigned across all participants. This might raise concerns relating to whether psychotherapy effects were genuine or attributable to psychotherapy-placebo effects. Finally, as a result of our study inclusion criteria, such as English language studies only and including outcomes studied by three studies without therapy, we excluded a large number of studies, including one with a large sample. This 
creates a selection bias and may raise concerns relating to the generalizability of the results.

\section{Conclusions}

Naltrexone treatment is efficacious in reducing alcohol consumption, but not reducing cravings. Adding psychotherapy on top naltrexone did not result in any significant additional benefit for AUD patients.

\section{Additional Information \\ Disclosures}

Human subjects: All authors have confirmed that this study did not involve human participants or tissue. Animal subjects: All authors have confirmed that this study did not involve animal subjects or tissue. Conflicts of interest: In compliance with the ICMJE uniform disclosure form, all authors declare the following: Payment/services info: All authors have declared that no financial support was received from any organization for the submitted work. Financial relationships: All authors have declared that they have no financial relationships at present or within the previous three years with any organizations that might have an interest in the submitted work. Other relationships: All authors have declared that there are no other relationships or activities that could appear to have influenced the submitted work.

\section{References}

1. Ahmadi J, Babaeebeigi M, Maany I, Porter J, Mohagheghzadeh M, Ahmadi N, Dehbozorgi G: Naltrexone for alcohol-dependent patients. Ir J Med Sci. 2004, 173:34-37. 10.1007/BF02914522

2. Anton RF, Moak DH, Latham P, et al.: Naltrexone combined with either cognitive behavioral or motivational enhancement therapy for alcohol dependence. J Clin Psychopharmacol. 2005, 25:349-357. 10.1097/01.jcp.0000172071.81258.04

3. Anton RF, O’Malley SS, Ciraulo DA, et al.: Combined pharmacotherapies and behavioral interventions for alcohol dependence: the COMBINE study: a randomized controlled trial. JAMA. 2006, 295:2003-2017. 10.1001/jama.295.17.2003

4. Anton RF, Moak DH, Waid LR, Latham PK, Malcolm RJ, Dias JK: Naltrexone and cognitive behavioral therapy for the treatment of outpatient alcoholics: results of a placebo-controlled trial. Focus. 2003, 1:183189. 10.1176/foc.1.2.183

5. O’Malley SS, Jaffe AJ, Chang G, Schottenfeld RS, Meyer RE, Rounsaville B: Naltrexone and coping skills therapy for alcohol dependence: a controlled study. Arch Gen Psychiatry. 1992, 49:881-887. 10.1001/archpsyc.1992.01820110045007

6. Oslin D, Liberto JG, O’Brien J, Krois S, Norbeck J: Naltrexone as an adjunctive treatment for older patients with alcohol dependence. Am J Geriatr Psychiatry. 1997, 5:324-332. 10.1097/00019442-199700540-00007

7. Petrakis IL, Poling J, Levinson C, Nich C, Carroll K, Rounsaville B: Naltrexone and disulfiram in patients with alcohol dependence and comorbid psychiatric disorders. Biol Psychiatry. 2005, 57:1128-1137. 10.1016/j.biopsych.2005.02.016

8. Volpicelli JR, Rhines KC, Rhines JS, Volpicelli LA, Alterman AI, O'Brien CP: Naltrexone and alcohol dependence: role of subject compliance. Arch Gen Psychiatry. 1997, 54:737-742. 10.1001/archpsyc.1997.01830200071010

9. Davidson D, Saha C, Scifres S, Fyffe J, O'Connor S, Selzer C: Naltrexone and brief counseling to reduce heavy drinking in hazardous drinkers. Addict Behav. 2004, 29:1253-1258. 10.1016/j.addbeh.2004.03.027

10. Kranzler HR, Modesto-Lowe V, Van Kirk J: Naltrexone vs. nefazodone for treatment of alcohol dependence: a placebo-controlled trial. Neuropsychopharmacology. 2000, 22:493-503. 10.1016/S0893-133X(99)00135-9

11. Monti PM, Rohsenow DJ, Swift RM, et al.: Naltrexone and cue exposure with coping and communication skills training for alcoholics: treatment process and 1-year outcomes. Alcohol Clin Exp Res. 2001, 25:16341647. 10.1111/j.1530-0277.2001.tb02170.x

12. O'Malley SS, Rounsaville BJ, Farren C, Namkoong K, Wu R, Robinson J, O'Connor PG: Initial and maintenance naltrexone treatment for alcohol dependence using primary care vs specialty care: a nested sequence of 3 randomized trials. Arch Intern Med. 2003, 163:1695-1704. 10.1001/archinte.163.14.1695

13. O'Malley SS, Robin RW, Levenson AL, et al.: Naltrexone alone and with sertraline for the treatment of alcohol dependence in Alaska natives and non-natives residing in rural settings: a randomized controlled trial. Alcohol Clin Exp Res. 2008, 32:1271-1283. 10.1111/j.1530-0277.2008.00682.x

14. Oslin DW: Treatment of late-life depression complicated by alcohol dependence . Am J Geriatr Psychiatry . 2005, 13:491-500. 10.1097/00019442-200506000-00008

15. Pettinati HM, O’Brien CP, Rabinowitz AR, Wortman SP, Oslin DW, Kampman KM, Dackis CA: The status of naltrexone in the treatment of alcohol dependence: specific effects on heavy drinking. J Clin Psychopharmacol. 2006, 26:610-625. 10.1097/01.jcp.0000245566.52401.20

16. Brown ES, Carmody TJ, Schmitz JM, Caetano R, Adinoff B, Swann AC, Rush AJ: A randomized, double-blind, placebo-controlled pilot study of naltrexone in outpatients with bipolar disorder and alcohol dependence. Alcohol Clin Exp Res. 2009, 33:1863-1869. 10.1111/j.1530-0277.2009.01024.x

17. Kranzler HR, Wesson DR, Billot L: Naltrexone depot for treatment of alcohol dependence: a multicenter, randomized, placebo-controlled clinical trial. Alcohol Clin Exp Res. 2004, 28:1051-1059. 10.1097/01.ALC.0000130804.08397.29

18. Pettinati HM, Oslin DW, Kampman KM, et al.: A double-blind, placebo-controlled trial combining sertraline and naltrexone for treating co-occurring depression and alcohol dependence. Am J Geriatr Psychiatry. 2010, 167:668-675. 10.1176/appi.ajp.2009.08060852

19. Baltieri DA, Daró FR, Ribeiro PL, De Andrade AG: Comparing topiramate with naltrexone in the treatment of alcohol dependence. Addiction. 2008, 103:2035-2044. 10.1111/j.1360-0443.2008.02355.x 
20. Guardia J, Caso C, Arias F, et al.: A double-blind, placebo-controlled study of naltrexone in the treatment of alcohol-dependence disorder: results from a multicenter clinical trial. Alcohol Clin Exp Res. 2002, 26:13811387. 10.1111/j.1530-0277.2002.tb02682.x

21. Kiefer F, Jahn H, Tarnaske T, et al.: Comparing and combining naltrexone and acamprosate in relapse prevention of alcoholism: a double-blind, placebo-controlled study. Arch Gen Psychiatry. 2003, 60:92-99. 10.1001/archpsyc.60.1.92

22. Morley KC, Teesson M, Reid SC, et al.: Naltrexone versus acamprosate in the treatment of alcohol dependence: a multi-centre, randomized, double-blind, placebo-controlled trial. Addiction. 2006, 101:14511462. 10.1111/j.1360-0443.2006.01555.x

23. Balldin J, Berglund M, Borg S, et al.: A 6-month controlled naltrexone study: combined effect with cognitive behavioral therapy in outpatient treatment of alcohol dependence. Alcohol Clin Exp Res. 2003, 27:11421149. 10.1097/01.ALC.0000075548.83053.A9

24. Chick J, Anton R, Checinski K, et al.: A multicentre, randomized, double-blind, placebo-controlled trial of naltrexone in the treatment of alcohol dependence or abuse. Alcohol Alcohol. 2000, 35:587-593. 10.1093/alcalc/35.6.587

25. Heinälä P, Alho H, Kiianmaa K, Lönnqvist J, Kuoppasalmi K, Sinclair J: Targeted use of naltrexone without prior detoxification in the treatment of alcohol dependence: a factorial double-blind, placebo-controlled trial. J Clin Psychopharmacol. 2001, 21:287-292.

26. Gastpar M, Bonnet U, Böning J, et al.: Lack of efficacy of naltrexone in the prevention of alcohol relapse: results from a German multicenter study. J Clin Psychopharmacol. 2002, 22:592-598.

27. Morris PLP, Hopwood M, Whelan G, et al.: Naltrexone for alcohol dependence: a randomized controlled trial. Addiction. 2001, 96:1565-1573. 10.1046/j.1360-0443.2001.961115654.x

28. Latt NC, Jurd S, Houseman J, Wutzke SE: Naltrexone in alcohol dependence: a randomised controlled trial of effectiveness in a standard clinical setting. Med J Aust. 2002, 176:530-534.

29. Toneatto T, Brands B, Selby P: A randomized, double-blind, placebo-controlled trial of naltrexone in the treatment of concurrent alcohol use disorder and pathological gambling. Am J Addict. 2009, 18:219-225.

30. Krystal JH, Cramer JA, Krol WF, Kirk GF: Naltrexone in the treatment of alcohol dependence . N Engl J Med. 2001, 345:1734-1739. 10.1056/NEJMoa011127 